\title{
Optimization of Inter and Intra-Cell Layout in Cellular Manufacturing Systems using Simulated Software
}

\author{
V. Dinesh Kumar, K. Arun Prasath
}

\begin{abstract}
In this article we discuss how to manage "The Cellular Manufacturing System" (CMS) and explained the use of the Group Technology and in what reason that permit crumbling an Assembling Frame work and how is its management easier way than entire manufacturing system. Secondly, we develop model design by single row intra cell layout in three ways, they are Linear $L$ shape $-S$ shape by using Automation Studio Software (ASS), and find the best layout among these two types of design using ASS with minimize the Inter and Intra cell movements in static and dynamic environment from model and choose for the exertion is critic by using ARENA simulation software to identify optimized layout under Inter and Intra - cell of both environment in CMS.
\end{abstract}

Keywords : Cellular Manufacturing System (CMS), Automation Studio Software (ASS), Group Technology GT, ARENA Simulation software, and Inter-Intra Cell Layout.

\section{INTRODUCTION}

The vast majority of uses of gathering innovation need Cellular Manufacturing System (CMS) that permits breaking down an assembling framework into sub - frameworks, which makes its administration improved simpler than the whole assembling framework. This model for work place theme and it became an inalienable piece of direct assembling frameworks. It based the standard of gathering innovation and approach that creates a variety of things with as small waste as could sensibly be normal. Since a phone is a gathering of workstations apparatus instruments or gear masterminded to make a smooth stream so groups of parts can be prepared dynamically from workstation to another without trusting that a clump will be finished or requiring extra taking care of between tasks: In straightforward way, $\mathrm{CM}$ gathering (s) together appliance and a little group of staffs coordinated by a group head, that is the reason all the work on an item or part can be a practiced in a similar cell killing assets that don't enhance the item. In this article we disclose a way to deal with programmed discovery and acknowledgment of signs from characteristic scene and its

Revised Manuscript Received on December 29, 2019.

* Correspondence Author

V.Dinesh Kumar*, Department of Mechanical Engineering, Kalasalingam Academy of Research and Education, Krishnankoil, India Email: dineshkumar@klu.ca.in

K.Arun Prasath, Department of Mechanical Engineering, Kalasalingam Academy of Research and Education, Krishnankoil, India

Email: arunprasath@klu.ac.in application to a sign interpretation task. The essential idea of $\mathrm{CM}$ is to diminish the span of the procedure and inventories to meet market response times. Some of them to configuration better component portrayals and model or both: We additionally got advantages space decrease, quality improvement; work cast decrease, and fundamental ability of apparatus usage. The point of this work is lean assembling is the constrained minimization of waste, and to achieve most extraordinary capability resources.

The major design for CM demand for three phases

a. Gathering of Parts

b. Production equipment in to cells

c. Task of apparatus cells to division inside the shop floor (bury cell or facility layout).layout of the machine inside every phone (intra-cell or apparatus format) in which the design chooses where to put every one of the offices, apparatuses, gear and staff in the assembling activity inside the cell and it decides the manner by which materials and different information sources course through the activity. Generally minor change in position of a machine in a generation line can influence the movement of materials essentially.

\section{SKETCH IDENTIFICATION AND PROBLEM DEFINITION}

We have two sketches, one is the office design in CMS includes the game plan of cells inside the floor space, in order to limit the entomb - cell format development, the other one is the machine design in CMS takes the course of action of apparatuses inside cells to limit the intra - cell development. There are three apparatus plans, which are recognized by Hassan M. m. D [1], they are

a. Solitary line format: in this design, various appliances are organized in a solitary column with regards to the succession of tasks. The sketch is taking various shapes like linear, semicircular or shape ' $u$ '.

b. Multi-row sketch: the appliances are arranged in many rows. The appliances in every line co-operates one another with the appliances in different lines.

c. Loop design: in this format: The appliances are master-minded in a way and the development of parts is generally unidirectional. The static machine cellular layout (SMCL) is intended to work on static quantitative demand.

But now, today's consumer market there is frequent changes in product mix and

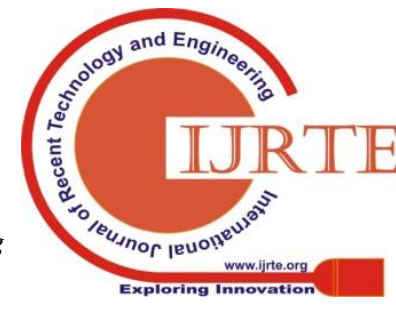


demands, which cannot be accommodated by SMCL.

We take the quantitative values of three period planning horizons given in table -1 for our calculation purposes. Note that earlier, the sketch problem is considered as static one. But today's consumer's market, there is frequent demand fluctuations, which cannot be accommodate by static - one. So that the implementation of cells could however, have some disadvantages as compared to traditional sketches. Implementing manufacturing cells needs high investment in appliance installation, results in lack of flexibility in handling the demand changes imbalanced utilization of appliance and labor etc., These mentioned disadvantages could however be turned positive by carefully identifying their causes and correcting them. Presently analysts proposed better model calculations to settle (Dynamic Layout Problem) DLP, Layout ID and issue definition [2] had clarified about concentrating on characteristics of multi-variety and little group making of electronic items, a workshop format advancement strategy dependent on cell producing is proposed. To begin with, the main arrangement and its issues are analyzed. By then, item amount assessment is performed and the item families are sorted. Centering at the item families, calculated amounts, non-strategic amounts are viewed as utilizing orderly format arranging (SLP) technique. Machine results show that the strategy is feasible and beneficial, and the calculated and non-calculated lists are better after headway [3] and appliance cell in a batch oriented production framework. This approach integrates a local search heuristic with a genetic algorithm. Using the genetic algorithm the set of appliance cells are generated. The motto is to form a set of appliance/part groups and improve it, if possible. So this approach is useful in cell formation in any batch oriented production framework [4] said that a poor sketch would bring about high work in process, longer holding up times, low productive planning and expanded material overseeing costs. Office format configuration decides course of action, area and dispersion of apparatuses in an assembling office to accomplish decrease in setting aside a few minutes, boost of efficiency as for generation plan. This paper centers around headway and reproduction of office format utilizing Merger Coefficient Formulae for a run of the mill office design [5] in this examination, preparing times and the frequencies of the parts are additionally considered. It is expected that the heap of every machine is known and is the augmentation of the handling times and frequencies. For this situation cells are shaped to accomplish the higher loads inside cells. The proposed model is about the situation when elective advances are accessible and the goal is to expand the heaps inside cells. Other than the new model, other primary commitment of this investigation is the computational examination. The results show that the new model is providing acceptable solution within the logical runtimes [6] this paper shows that considering the interrelationship among manufacturing functions will not only increase productivity but also helps to simplify the manufacturing process. Specifically, an alternate process planning approach has been developed by considering and demonstrating the interrelationship between two of the three major functions of manufacturing (process planning and GT). These specifications are based on the individual capabilities of the appliances rather than their types [7-8]

conveyed that in cellular manufacturing. In this paper, another scientific technique for CMS considering different creation factors including elective procedure routings and machine dependability with stochastic appearance and administration times in a powerful situation is proposed. Additionally in view of the unpredictability of the given issue, a Benders' disintegration approach is applied to take care of the issue proficiently; Auxetic structures display negative Poisson's proportions in at least one bearings. At the point when extended, they will become fatter or more slender when compacted, as opposed to traditional materials (like elastic, glass, metals, and so forth.) toward the path ordinary to the applied stacking heading. The present work expects to create diverse thickness auxetic cell structures break down their distinctive mechanical properties and features the von-misses stresses create during pressure and strain of these auxetic cell structures [9] in this paper the issue is more mind boggling than expected booking issues in that we register extra time prerequisites and settle on planning choices at the same time. Scientific Model 2 was the best numerical model as for both benefit and execution time. This model thought about incomplete additional time periods and furthermore permitted diverse extra time periods on cells. Therefore, these models can be utilized to settle on these choices in numerous modern settings [10] clarified about customary machining of cell metals prompts infelicitous surface harm and poor exactness. Besides, the chip development and the framework portrayal of the surface harm are as yet obscure. Microscopic limited component model will address the impact of the cutting parameter and apparatus geometry on the chip arrangement process, just as certain plans to improve the machining of cell metals [11] conveyed that Business process Re-Engineering (BPR) takes the Radical Redesigning unpredictable and dynamic procedures in Research and advancement (R\&D) associations with multi-layer ventures is a strenuous errand. In this article our aims in developing the models to determine the complete expense, a sum of material managing expense for the three planning horizon for five appliances combination assigned for various operations, when the appliances are assigned in a row of a cellular fabricating framework, we have taken the material managing and relocation expense values and their results of dynamic and static comparing environments to give optimum single row sketch in static environment. From the objectives of various papers and gathering information were given a correct view on problems in sketch and helps to solve the formulated model with good point of results with the help of software. And we will focus on applying the software to find optimum sketch by the assumptions (from our references) required for solving the problem $t$ follows:

1) First, cell development is achieved, that is the appliance belongs to which cell is known.

2) Size of any appliance area sector (x, y) can accommodate the prodigious appliance in that specific cell.

3) An appliance is relegated to any area.

4) Stacking and emptying at the focal point of each appliance.

5) Cell distance between two cells is equal. 
6) Distance to travel is assessed by estimating the rectilinear good ways from focus of the appliance to the focal point of the goal appliance.

The principle target of this model is to limit the absolute expense in a planning horizon and defined as a sum of Material managing expense. Also find the optimal single row sketch in static environment.

\section{MATERIAL MANAGING EXPENSE}

The material managing cost depends on parts to be moved between appliances according to the series of operation. The material movement is calculated using Manhattan method. Material handling cost,

$$
\xi=\Psi \times \omega
$$

Where,

$\xi=$ Material handling cost $=$ Total traveling distance, $\omega=$ Unit cost for traveling score, $\Psi=$ price per unit appliance displacement.

\section{NUMERICAL ILLUSTRATION}

The application of GT to production frameworks has in many firms led to the introduction of CM. This paper studies the changes that are required in the organization of the planning and control frameworks when applying CM. We review existing structure for designing such a planning and control framework and propose a new framework that gives attention to decisions with respect to the aggregation and abstraction of information on resources, orders and time. We discuss various contributions from previous scholar's results, the applicability of well-known approaches of planning and control to CM to illustrate the model, a numerical example is shown below. Grouping the appliances and parts in a CMS based on similarities is known as the cell formation. We proposes a method for design of cell sketch for a fabricating framework under dynamic demand environment for automation batch production industry the performance of a production framework depends not only on the quality of the decomposition of the framework in cells and departments, but also on the quality of the production planning framework that is being used to plan and control the flow of work. However, the goodness of fit between both frameworks is of the greatest importance to take full advantage of the benefits of cellular manufacturing. The design of the production planning and control framework should meet the necessity of the production framework. The issue takes 5 parts that are processed in 9appliances in that they find minimum three sequence of operations. The details about the operational sequence quantitative demands and Appliance rearrangement cost in a 3 period planning horizon. And the optimized value methods are calculated using the simulation software was tabulated below. In this cluster of tables, the table [2] gives the result of optimized linear single row sketch and the table [3] shows the weighing up of linear and L shape sketch, the optimized values. This would not take care of the issues referenced up until this point. In this way, we need to investigate the structure of production planning and control frameworks that can be applied in cell fabricating various audit articles on production control in cellular manufacturing have appeared, The realization of benefits expected from cellular manufacturing largely depends on how effectively the stages of the design have been performed Cellular Manufacturing. Major advantages, including reduction in lead times and work-in-process inventories and reduction of setup times due to similarity of part types produced the Part-family and appliance-Cell formation with the consideration of duplicated appliances in a manufacturing cell design is proposed in this research. In existing methods, part families and appliance cells are firstly determined. Then, the incidence matrix of the cell formation is reconsidered for assignment of the duplicated appliances. These ways are difficult and complicated. Moreover they do not guarantee for effective solutions and problems are discussed here, Also we use some denotations to identify the appliance names and its placement over their working allotments they are "M1" here the M represents the appliance number in the suffix the numerical were explained according to their working plan.

Table- I: Quantitative values in static environment sketch

\begin{tabular}{|c|c|c|c|}
\hline Parts & Period 1 & Period 2 & Period 3 \\
\hline 1 & 10 & 35 & 90 \\
\hline 2 & 30 & 50 & 25 \\
\hline 3 & 45 & 15 & 40 \\
\hline 4 & 70 & 80 & 55 \\
\hline 5 & 85 & 60 & 70 \\
\hline
\end{tabular}

Table- II: Comparisons between L shape row sketches

\begin{tabular}{|c|c|c|c|}
\hline $\begin{array}{c}\text { Serial } \\
\text { Number }\end{array}$ & $\begin{array}{c}\text { Sequence of } \\
\text { Sketch }\end{array}$ & $\begin{array}{c}\text { From } \\
\text { paper } \\
\text { (Total. } \\
\text { Cost in Rs) }\end{array}$ & $\begin{array}{c}\text { Using Software } \\
\text { (Total. Cost in } \\
\text { Rs) }\end{array}$ \\
\hline 1 & 176542893 & 4480 & 4295 \\
\hline 2 & 146275839 & 4410 & 4218 \\
\hline 3 & 135879264 & 4940 & 4125 \\
\hline
\end{tabular}

Table- III: Comparisons between linear \& S shape single row sketches

\begin{tabular}{|c|c|c|c|}
\hline $\begin{array}{c}\text { Serial } \\
\text { Number }\end{array}$ & $\begin{array}{c}\text { Sequence of } \\
\text { Sketch }\end{array}$ & $\begin{array}{c}\text { From } \\
\text { paper } \\
\text { (Total. } \\
\text { Cost in Rs) }\end{array}$ & $\begin{array}{c}\text { Using Software } \\
\text { (Total. Cost in } \\
\text { Rs) }\end{array}$ \\
\hline 1 & 176542893 & 4480 & 4502 \\
\hline 2 & 146275839 & 4410 & 4484 \\
\hline 3 & 135879264 & 4940 & 5197 \\
\hline
\end{tabular}

\section{V.RESULT AND DISCUSSION}

We have considered the optimum sketch sequence with the order for various parts and number of days is given as inputs. The optimal arrangement of appliances and traveling distances for each part in linear single row and multi row sketches are obtained. And finally compare the results with process sequence and quantitative demand for various timing are given as inputs. The optimal arrangement of appliances and traveling distances for each day in a row sketch is obtained. Material managing price relies upon parts to be moved between appliances as indicated by the sequence of operation. But the transport expense depends only on the movement and flow of materials in a working sketch. We have considered the optimum sketch sequence with the order for various parts and no of days are given as inputs. The optimal arrangement of 
appliances and traveling distances for each part in linear single row are obtained. And finally compare the results obtained using software from table - II the travelled distance and their cost for travel in linear row sketch is too high and we obtained the optimum cost using the software, similarly table - III deals with the comparison of linear and L shape sketch gives the value to compare the linear and sketches and frameworks into manufacturing plants', 'scaled down organizations' or 'cells' with clear clients, obligations and significant components in abusing the advantages of $\mathrm{CM}$ is proficient sketch designs. Some problems expressed by the company were large and needless volume of shop, material handling price, difficulties and confusion over production planning, long duration products lead times resulting in losing customers and high overhead costs The primary reason that assembling companies are pulled in towards implementing CM is that the benefits of CM can normally be acknowledged with relatively low capital investment by relocating and conceivably copying certain appliances instead of other mechanized techniques used for design not for analyzing the work. The comparison of the implemented solution algorithm with the conventional mixed integer linear and mixed integer nonlinear models verifies the efficiency of Benders' disintegrating approach especially in terms of computation time. [12] he considers a Lean Manufacturing (LM) is considered as a rapid growing manufacturing culture. Lean Manufacturing might be characterized as the procedure which is utilized for the expulsion of a wide range of waste in the creation procedure to improve the proficiency. The essential aggressive proportion of lean is "ability to react to the client and fulfill his prerequisites by making the procedure of the creation effective and squander free [13].

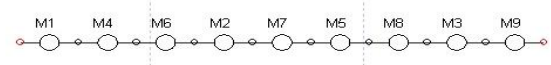

Fig. 1. Automation studio's linear sketch model

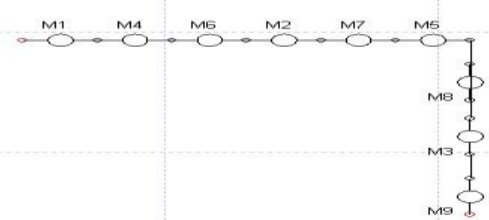

Fig. 2. Automation studio's L shape sketch model

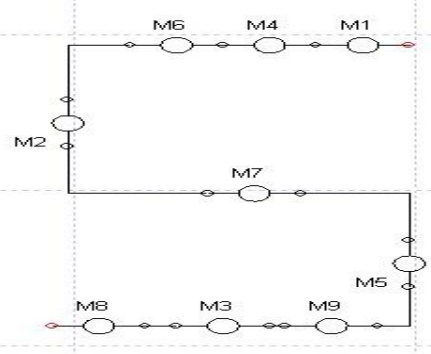

Fig. 3. Automation studio's S shape sketch model

From above comparison using data and software simulation the optimum single row sketch by price is linear single row sketch. In table: 3 give the optimum sequence of sketch in linear movements of appliances. ] we develop a Bayesian

semi parametric approach to GARCH-type models. The innovations follow the class of scale mixtures of Gaussian distributions with Dirichlet process prior in the mixing distribution. It is also shown how to undertake Bayesian prediction of the Value at Risk (VaR). The performance of the proposed semi parametric method is illustrated by utilizing mimicked and genuine information from the Hang Seng Index (HSI) and Bombay Stock Exchange index (BSE30) [14] similarly shapes sketch price.

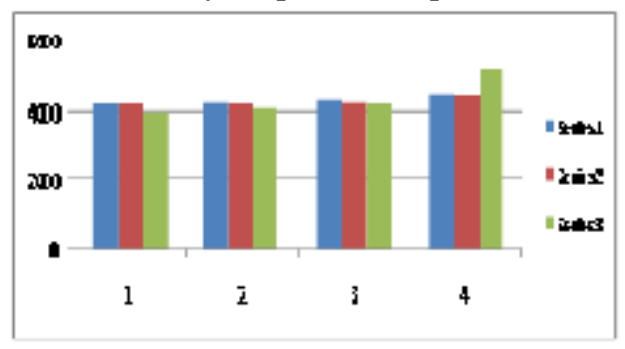

Fig. 4. Comparison of linear with L shape, U shape and S shape layouts

The above column chart gives the comparison between three sketches of three planning period horizon. From graph the red bar indicates the $\mathrm{L}$ shape sketch the blue color indicates the U-shape sketch similarly the green color indicates the S shape sketch. In this graph we easily find the optimum sketch with minimum transportation cost. And the $\mathrm{Y}$ axis arranged with the values of linear single row sketch and $\mathrm{X}$ with desired values of each values obtained through software.
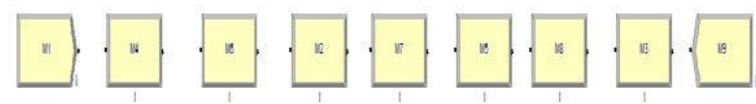

Fig. 5 Model of an Optimized linear sketch design

This above arena mode of linear sketch shows the linear arrangement of 146275839 operational sequences which has only minimum travelling distance and material handling cost. Also here the total cost is about Rs4270, from these wrapping up we got in the final result through both software and manual the arena dialogue box starts with the notations "m1" to the optimized order and ends with "M9".

\section{CONCLUSION}

Sketch determines the way in which materials and various inputs (like people and information) flow through the process. Generally little changes in the locale of an appliance in a factory can affect the flow of materials considerably. This in turn can affect the expenses and proficiency of the overall manufacturing operation. Failing can lead to inefficiency, inflexibility, huge volumes of inventory and work in progress, high costs and unhappy clients. Appliance sketch with three fundamental classification is identified and we calculate the transportation cost for single row sketch in that especially linear, $\mathrm{S}$ shape and L shape using ARENA software. Transportation cost comparison between the sketches in both the working environment using ARENA simulation software. Some in this paper we proposed to present a meticulous case study in a 
process industry to study the existing sketch design simulation software. In that we made the comparison and find that the linear sketch has minimum transportation cost than L shape, following that ascending with S shape sketch. From references and software values,

the optimum sketch by cost is linear single row sketch in static environment because linear arrangement of appliances without any backtracking movements may improve productivity and reduce cost of transportation makes this linear sketch as best in cellular fabricating frameworks. And the analyzing the movements and reduction in cost using a software is very simple from our literature survey comes factual so, we have planned to develop a model and also with minimum. We have designed the ideal sketch considering various sketches with an aim of minimizing the expense by reducing the total travelling distance of materials and change the existing sketch design for selected process industry. This optimum sketch design is executed using ARENA simulation software with different form of appliance sketches.

\section{REFERENCES}

1. Hassan.M.D, "Application layout problem in modern manufacturing process" Journal of Engineering, 2015, pp.15-24.

2. Nazir, Aamer, and Jeng-Ywan Jeng. "Buckling behavior of additively manufactured cellular columns: Experimental and simulation validation." Materials \& Design, 2015, pp. 10-25.

3. Soto, Ricardo, "Solving the manufacturing cell design problem through binary cat swarm optimization with dynamic mixture ratios", Computational intelligence and neuro science, 2019, pp.20-32.

4. Bortolini, Marco, Francesco Gabriele Galizia, and Cristina Mora. "Dynamic design and management of reconfigurable manufacturing systems." Procedia Manufacturing 33, 2019, pp. 67-74.

5. Dey,Swati, "Data-Driven Design Optimization for Industrial Products". Optimization in Industry, 2019. pp. 253-267.

6. Rustam, Zuherman, and Rahmat Hidayat, "Indonesia Composite Index Prediction using Fuzzy Support Vector Regression with Fisher Score Feature Selection",International Journal on Advanced Science, Engineering and Information Technology,2019,pp. 121-128.

7. Rupp, Markus, "Predatory journals: a major threat in orthopaedic research" International ortho poetics, 2019, pp.509-517.

8. Lee, T., and Y. Loong. "A review of scheduling problem and resolution methods in flexible flow shop," International Journal of Industrial Engineering Computations, 2019, pp. 67-88.

9. Sánchez, Alberto, "Introduction of building information modeling in industrial engineering education: Students' perception", Applied Sciences, 2019, pp.32-87.

10. Husain, Sadang ,"Combustion Properties of Briquette from Halaban (vitex pubescens vahl) Charcoal, Bottom Ash and Fly Ash," International Journal of Chem Tech Research, 2019.

11. Belhouchet, Khaled, Abdelhafid Bayadi, and M. Elhadi Bendib. "Artificial neural networks and genetic algorithm modelling and identification of arc parameter in insulators flashover voltage and leakage current," International Journal of Computer Aided Engineering and Technology, 2019, p. p. 1-13.

12. Debnath, Saswati, and Pinki Roy, "Study of speech enabled healthcare technology", International Journal of Medical Engineering and Informatics, 2019, p. p.71-85.

13. Singla, Anuj, Inderpreet Singh Ahuja, and Amanpreet Singh Sethi, "An evaluation of status of technology push and demand pull practices for sustainable development in manufacturing industries", International Journal of Technology, Policy and Management, 2019, p.p.32-71.

14. Wognum, Nel,"Transdisciplinary systems engineering: implications, challenges and research agenda", International Journal of Agile Systems and Management, 2019, p.p.58-89.

\section{AUTHORS PROFILE}

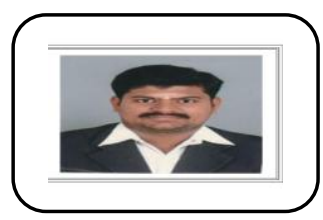

V.DineshKumar, an Assistant Professor in the Department of Mechanical Engineering, Kalasalingam Academy of Research and Education, Krishnankoil .He has experienced in the area of operational research and thermal conductivity.

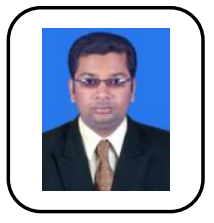

K. ArunPrasath, working as an Assistant Professor in the Department of Mechanical Engineering ,Kalasalingam Academy of Research and Education, Krishnankoil. He has research experience in the field of cellular manufacturing and published some good works in reputed journals. And also his research extend to composite materials for low velocity applications 